\section{Genetic biomarker trumps tissue type in landmark oncology approval}

The FDA granted accelerated approval to Merck \& Co.'s PD1 blocker pembrolizumab for all cancers with a specific genetic biomarker, regardless of which tissue type is involved. "This is an important first for the cancer community," said the FDA's Richard Pazdur, acting director of the Office of Hematology and Oncology Products. "Until now, the FDA has approved cancer treatments based on where in the body the cancer started."

The FDA has already approved pembrolizumab for several tissue-dependent indications, including melanoma, lung cancer and head and neck cancer. With the new supplementary approval, oncologists can now prescribe the checkpoint inhibitor for patients with any cancer type that has high microsatellite instability (MSI-H) or mismatch repair deficient (dMMR) tumours.

MSI-H and dMMR tumours carry genetic mutations that prevent proper DNA repair, leading to high mutational loads and increased production of mutated proteins that can act as antigens to activate the immune system. Drug developers hypothesized that checkpoint inhibitors such as pembrolizumab that stimulate the immune system might consequently be particularly effective in these cancers. When Merck administered its antibody to $149 \mathrm{MSI}-\mathrm{H}$ or dMMR patients - with 15 different cancer types, from 5 different clinical trials - treatment led to a complete or partial response rate of $40 \%$.

Whereas Merck's initial development of pembrolizumab relied on a conventional tissue-dependent development programme, a few firms are now developing novel drugs under a tissue-agnostic approach. Loxo Oncology's tropomyosin receptor kinase (TRK) inhibitor larotrectinib could be the first of these to make it to market. In a preliminary analysis of 55 cancer patients who were treated on the basis of TRK fusions rather than tissue type, larotrectinib treatment led to a $\underline{76 \%}$ confirmed objective response rate. The company plans to file for tissue-agnostic FDA approval by late 2017 or early 2018.

Ignyta is pursuing a similar strategy for its phase II TRK inhibitor entrectinib. Other companies that are embracing the tissue-agnostic approach include TP Therapeutics and Blueprint Medicines.

Asher Mullard

\section{Finding fault with Bial's fatal FAAH inhibitor}

In January 2016, a phase I trial of Bial's oral analgesic BIA 10-2474 killed one volunteer and caused brain damage in four others. The fatal effects of the fatty acid amide hydrolase (FAAH) inhibitor may have been caused by off-target activity on several lipases, an international team of researchers now report (Science 356, 1084-1087; 2017).

When the tragedy first occurred, researchers speculated on three general possibilities for why BIA 10-2474 was toxic: errors may have occurred in the manufacturing, handling or administration of the drug; on-target FAAH activity may have led to overstimulation of certain signalling pathways; or off-target activity may have triggered the side effects. Regulatory authorities dismissed the first possibility, and Pfizer's FAAH inhibitor PF-04457845 made it through phase II trials before failing on efficacy rather than safety, calling into question the on-target toxicity.
To understand whether the off-target activity profile of BIA 10-2474 was to blame, researchers used an activity-based proteomic method to look at what other targets the drug binds to in human cells. Several serine hydrolases that are not targeted by PF- 04457845 topped the list, they report. Many of these hydrolases modulate cellular lipid metabolism and show substantial expression in the brain. These findings raise "the possibility that disruption of cellular lipid networks may have contributed to the compound's neurotoxicity," the authors write.

The study did not assess the off-target activity of Johnson \& Johnson's experimental FAAH inhibitor JNJ-42165279, which the company put on clinical hold after the BIA 10-2474 disaster.

"The study highlights the general utility of [activity-based proteomic methods] as a versatile chemical proteomic method to assess on-target engagement and off-target activity of covalent drugs to guide therapeutic development," the authors conclude.

Asher Mullard

\section{Biotech RED spending continues to rise}

Biotech RED spending hit US\$45.7 billion in 2016, up 12\% from 2015, found an annual analysis of the sector by consulting firm EY (see FIG. 1). This was the seventh consecutive year that these firms increased their cumulative R\&D spending, and the second consecutive year in which RED spending growth outpaced revenue growth (7\%).

US firms poured the most new money into $R \& D$, increasing their investment in science by $14 \%$. European firms spent only an extra $3 \%$ on RED in 2016.

“The biotech industry's financial commitment to R\&D, while impressive, needs to be coupled with efficiency improvements to achieve better returns and ultimately to drive greater affordability of its products," said Pamela Spence, EY Global Life Sciences Leader. "With pricing pressures expected to escalate, firms will need to incorporate new digital and artificial intelligence technologies into their traditional drug target selection and overall RED processes to achieve those returns or risk being outdone by firms that do."

Asher Mullard

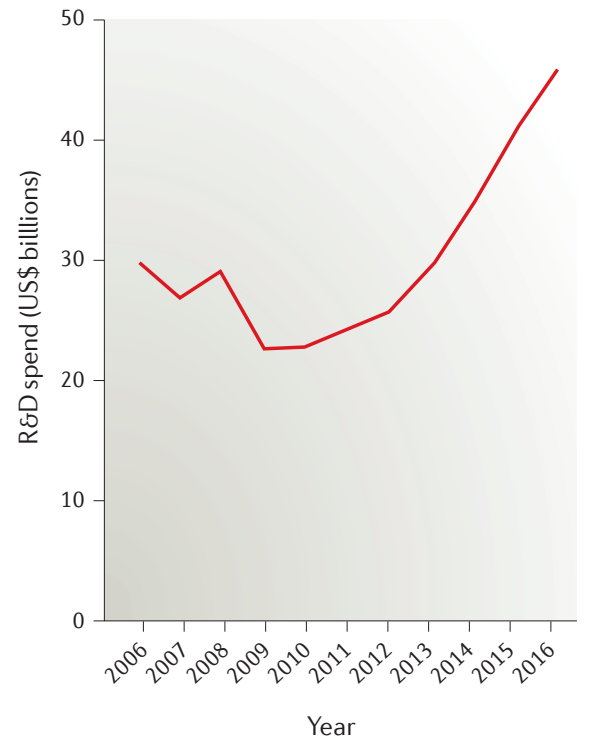

Figure 1 | Biotech RED spending data from the EY Biotechnology Reports 2008-2017. When annual reports provided inconsistent RED spending data, data from the latest report were used. The most recent analysis studied 708 biotech firms, and excluded large pharmaceutical companies. 